\title{
Short-Term Fire Effects on Small Mammal Populations and Vegetation of the Northern Chihuahuan Desert
}

\author{
Tony J. Monasmith, ${ }^{1}$ Stephen Demarais, ${ }^{2}$ J. Jeffrey Root, ${ }^{1}$ and Carlton M. Britton' \\ ${ }^{1}$ Department of Natural Resources, Texas Tech University, Lubbock, TX 79409, USA \\ ${ }^{2}$ Department of Wildlife, Fisheries and Aquaculture, Mississippi State University, Starkville, MS 39762, USA
}

Correspondence should be addressed to Stephen Demarais, sdemarais@cfr.msstate.edu

Received 6 January 2010; Revised 26 June 2010; Accepted 14 September 2010

Academic Editor: Jean Clobert

Copyright ( 12010 Tony J. Monasmith et al. This is an open access article distributed under the Creative Commons Attribution License, which permits unrestricted use, distribution, and reproduction in any medium, provided the original work is properly cited.

\begin{abstract}
Fire is an important ecological factor in semidesert grass-shrub community dynamics, but there is a lack of designed field experiments documenting effects on vegetation and small mammals. We document effects of June prescribed fire on vegetation and small mammals on 20, 25-ha study areas in the Northern Chihuahuan Desert of Southern New Mexico, USA one month and one year posttreatment. Canopy cover of shrubs and grasses recovered to 68 and $27 \%$ of the preburn canopy cover, respectively, after one year. Prescribed burns during June enhanced short-term forb production by reducing competition from grasses and shrubs. Thirty thousand trap-nights yielded 1744 captures of 766 individuals of 15 small mammal species. Burns did not affect small mammal species richness and species diversity. Relative abundance of Merriam's kangaroo rats (Dipodomys merriami) was $91 \%$ greater on burned sites than on control sites one year postburn. Silky pocket mouse (Perognathus flavus) relative abundance was $221 \%$ greater on burned sites one year postburn. Chihuahuan Desert pocket mice (Chaetodipus eremicus) responded negatively to the fire, with relative abundance $170 \%$ greater on control sites $(P=.080)$. Burning produced short-term benefits for two heteromyids, Merriam's kangaroo rats and silky pocket mice.
\end{abstract}

\section{Introduction}

Historically, fire may have played a role in defining the composition of semidesert grass-shrub vegetation types [1], such as the Northern Chihuahuan Desert ecosystem (NCD). Fire, rainfall, grazing pressure, and seed bank resources may interact to influence small mammal communities [2]. Fire can impact small mammals directly, such as with increased mortality [3], and indirectly, by affecting selection and use of microhabitats affected by fire [4]. Such impacts on small mammals could have potential long-term impacts on vegetation, as kangaroo rats (Dipodomys spp.) may increase the persistence of shrublands through graminivory [5].

Studies have documented small mammal responses to fire in shrub and grassland habitats but typically lack spatial replication [6-8] or presampling [9]. Without a prior index of population levels on burned areas, results could misrepresent changes in diversity or assemblages of small mammal populations [10]. Interest in the role of fire in ecological communities [11] has spurred the development of a priori studies with spatial replication and random assignment of treatments, but most have been in forested systems [12-14].

Few studies have documented the effects of fire on vegetation and small mammal communities in desert habitats. C. E. Bock and J. H. Bock [7] examined three Arizona wildfires in a sacaton (Sporobolus spp.) grassland and attributed small mammal community shifts to decreased total green vegetation and increased seed-producing plants. Following a June prescribed fire in the Sonoran Desert, a decreased density of the small mammal community was attributed to fire mortality and elimination of cover and nesting materials [15]. A wildfire in the Simpson Desert in Australia decreased small mammal species richness and abundance of the desert mouse (Pseudomys desertor) due to a loss of habitat and increased exposure to predation [16]. A landscapelevel study evaluating impacts 0 to $>25$ years postfire in desert grasslands of Australia related variable small mammal 
assemblages to locality, rainfall, seed bank resources, and cattle grazing [2].

Effects of fire on vegetation and small mammals in desert habitats have received little attention, and studies of effects of fire in the NCD are largely lacking. Our objective was to document short-term influences of a growing season (June) prescribed fire on species diversity, richness, and relative abundance of small mammals and on vegetative cover, shrub density, and frequency of grasses and forbs in the creosote/tarbush community of the NCD.

\section{Study Area}

We conducted this study in the NCD, on Fort Bliss Military Reservation, Otero County, New Mexico. Study sites lay in the transition zone between the Tularosa Basin to the south and the Sacramento Mountains to the north, with an average elevation of $1289 \mathrm{~m}$. The topography was nearly level with Mimbres and Tome soil associations on the bottom lands and alluvial fans, respectively. The region was characterized as arid to semiarid with variable precipitation of $8-25 \mathrm{~cm}$ of rainfall annually, with greater than $70 \%$ occurring during May-October [17]. Cattle had not grazed the study sites for over 50 years.

The shrub community was dominated by creosote bush (Larrea tridentata) and tarbush (Flourensia cernua) but included honey mesquite (Prosopis glandulosa), fourwing saltbush (Atriplex canescens), wolf-berry (Lycium berlandieri), soaptree yucca (Yucca elata), Spanish dagger (Yucca torreyi), and various Opuntia spp. The dominant grass and forb species on the sites were bush muhly (Muhlenbergia porteri) and Gregg's heliotrope (Heliotropium greggii), respectively. Other common species included burrograss (Scleropogon brevifolius), black grama (Bouteloua eriopoda), ear muhly (i.e., sand muhly, Muhlenbergia arenacea), and silverleaf nightshade (Solanum elaegnifolium).

\section{Methods}

Twenty 25 -ha study sites $(500 \times 500$-m) were selected during January 1995. We conducted step-point counts [18] across each site to determine shrub frequency, prevalence of surface rocks, and availability of fine fuel to carry a fire. To account for minor elevation, soil, and vegetative variation across the landscape, we paired the sites based on similarities of the above criteria. We randomly assigned treatments (treatment = burned, control $=$ unburned) within each pair (i.e., block).

We placed perimeter black lines $(30-35 \mathrm{~m})$ around each of the burn treatment sites during 20-27 March, 1995, to ensure an adequate safety margin during head fire application. We burned the black lines under the following mean environmental conditions: temperature $19^{\circ} \mathrm{C}$, humidity $33 \%$, and wind speed $9 \mathrm{~km} / \mathrm{hr}$. We burned the treatment sites during 13-15 June, 1995, using the strip head fire technique to increase ignition coverage [1]. A relatively hot fire was required to carry the fire across the light, discontinuous fuels associated with the vegetative community. We burned the strip head fires under the following environmental conditions: temperature $36^{\circ} \mathrm{C}$, humidity $14 \%$, and wind speed $11 \mathrm{~km} / \mathrm{hr}$.

We sampled vegetation prior to treatment application during May 1995 and posttreatment during 5-30 July, 1995 and 5-30 July, 1996, on six randomly placed 45$\mathrm{m}$ line transects on each site. We used continuous line intercept methodology to estimate canopy cover percentage of grasses, forbs, and shrubs. Bare ground (the amount of soil without detritus coverage) was also measured along each line transect. We estimated shrub and cacti densities within a 2-m belt transect around each of the 6 line transects. Herbaceous species frequency was estimated within $5,1 / 4-$ $\times 4$-m quadrats located randomly along each transect but only during preburn and one year postburn. Frequency was the percent of 30 quadrats per site which contained a given species.

We estimated small mammal species richness, diversity, and relative abundance (minimum number known alive) within each of the 20 study sites during 10-30 May, 1995 (pre-treatment), 10-30 July, 1995 (one month posttreatment), and 10-30 July, 1996 (one year posttreatment). A more prolonged trapping effort needed for estimating density was not possible because the large number of experimental units had to be sampled during a relatively brief summer sampling period. Treated and control sites within each block were trapped simultaneously. We sampled a 10 $\times 10$ trapping grid at the center of each study site with one Sherman live trap located at each of 100 trap stations spaced every $10 \mathrm{~m}$ for five consecutive nights, generating 500 trap nights per sampling event on each site. We placed unbaited traps at each station for one night prior to trapping to familiarize the animals with foreign objects in their home range [19]. We baited traps with oatmeal within three hours of sunset and checked them the following morning within four hours of daybreak to minimize heat-related mortality. We marked animals with numbered ear tags from National Band \& Tag Company, with the exception of pocket mice (Perognathus and Chaetodipus) which were toe clipped. Handling procedures were approved by the Texas Tech University Animal Care and Use Committee.

We estimated small mammal species diversity with Shannon's and Simpson's diversity indices [20, 21]. We also assessed species richness with the count of individual species present on a plot during sampling periods.

We analyzed results as a completely randomized block design with repeated measures, and the 20 study sites were the experimental units. The presample was used as a covariate if there was potential for pretreatment differences between sites $(P<.10)$. This alpha level was used as a conservative approach to reduce the probability of a type II error, increasing the likelihood that potential preexisting conditions were accounted for within our statistical analyses. Normality was assessed with the Shapiro-Wilk test, and in the event normality was violated, data were square-root transformed. If transformations were still nonnormal, we rank-transformed the data and tested the ranks with the above-defined analysis of variance [22]. Grass and forb frequency data were analyzed within years (1995 preburn and 1996 postburn) as a 1-way randomized block design analysis 
TABLE 1: Mean total shrub, grass, and forb canopy cover (\%) and bare ground (\%) prior to burning, May 1995 (PRE), one month postburn, July 1995 (POST95), and one year postburn, July 1996 (POST96), on 10 pairs of burned (B) and control (C) sites on Fort Bliss Military Reservation, Otero County, New Mexico.

\begin{tabular}{|c|c|c|c|c|c|c|c|c|c|}
\hline \multirow{2}{*}{ Cover } & & \multicolumn{2}{|c|}{ PRE } & \multicolumn{2}{|c|}{ POST95 } & \multicolumn{2}{|c|}{ POST96 } & \multicolumn{2}{|c|}{$P$} \\
\hline & & $\mathrm{B}$ & $\mathrm{C}$ & B & $\mathrm{C}$ & B & $\mathrm{C}$ & Burn & Time \\
\hline \multirow[t]{2}{*}{ Shrub } & $\bar{x}$ & 22.7 & 32.2 & 14.4 & 26.7 & 15.4 & 29.2 & .006 & .040 \\
\hline & $\mathrm{SE}$ & 1.6 & 10.3 & 1.9 & 1.9 & 2.5 & 2.2 & & \\
\hline \multirow[t]{2}{*}{ Grass } & $\bar{x}$ & 35.8 & 36.7 & $5.6 \mathrm{~A}$ & $36.8 \mathrm{~B}$ & $9.8 \mathrm{~A}$ & $35.0 \mathrm{~B}$ & 2 & \\
\hline & SE & 2.5 & 2.1 & 1.1 & 2.1 & 1.4 & 2.3 & & \\
\hline \multirow[t]{2}{*}{ Forb $^{1}$} & $\bar{x}$ & 1.7 & 2.5 & $2.0 \mathrm{~A}$ & $0.9 \mathrm{~A}$ & $19.3 \mathrm{~A}$ & $4.0 \mathrm{~B}$ & 2 & \\
\hline & SE & 0.3 & 0.6 & 0.4 & 0.5 & 2.7 & 0.8 & & \\
\hline \multirow[t]{2}{*}{ Bare ground } & $\bar{x}$ & 49.3 & 48.8 & $82.2 \mathrm{~A}$ & $51.5 \mathrm{~B}$ & $62.6 \mathrm{~A}$ & $52.0 \mathrm{~A}$ & 2 & \\
\hline & SE & 2.7 & 2.1 & 4.2 & 2.3 & 5.0 & 2.0 & & \\
\hline
\end{tabular}

${ }^{1}$ May 1995 results used as a covariate for postburn comparisons due to the potential for pretreatment effects $(.05<P<.10)$. Postburn results are covariateadjusted means.

${ }^{2}$ Treatment $\times$ time interaction $(P<.05)$. Means followed by same letter within years are not different $(P>.05)$.

TABle 2: Density (\#/ha) of shrub and cactus species prior to burning, May 1995 (PRE), one month post burn, July 1995 (POST95), and one year post burn, July 1996 (POST96), on burned (B) and control (C) sites on Fort Bliss Military Reservation, Otero County, New Mexico.

\begin{tabular}{|c|c|c|c|c|c|c|c|c|c|}
\hline \multirow{2}{*}{ Species } & & \multicolumn{2}{|c|}{ PRE } & \multicolumn{2}{|c|}{ POST95 } & \multicolumn{2}{|c|}{ POST96 } & \multicolumn{2}{|c|}{$P$} \\
\hline & & B & $\mathrm{C}$ & B & $\mathrm{C}$ & B & $\mathrm{C}$ & Burn & Time \\
\hline Atriplex ${ }^{1}$ & $\bar{x}$ & 20.4 & 21.3 & 2.8 & 24.1 & 0.9 & 18.5 & .075 & .099 \\
\hline canescens & SE & 6.0 & 8.5 & 2.0 & 12.9 & 0.9 & 11.0 & & \\
\hline Flourensia & $\bar{x}$ & 1000.0 & 790.7 & $219.4 \mathrm{~A}$ & $851.9 \mathrm{~B}$ & $263.0 \mathrm{~A}$ & $794.4 \mathrm{~B}$ & 2 & \\
\hline сеrnua & SE & 318.4 & 241.2 & 99.1 & 258.2 & 107.5 & 248.8 & & \\
\hline Larrea & $\bar{x}$ & 3050.0 & 2735.2 & 482.4 & 2685.2 & 781.8 & 2779.6 & $\leq .001$ & .032 \\
\hline tridentata & SE & 248.8 & 194.0 & 130.3 & 230.4 & 203.6 & 269.7 & & \\
\hline Parthenium & $\bar{x}$ & 102.8 & 64.8 & 26.9 & 61.1 & 61.1 & 55.6 & .746 & .131 \\
\hline incanum & SE & 58.8 & 51.1 & 14.8 & 48.4 & 32.2 & 42.9 & & \\
\hline Prosopis & $\bar{x}$ & 17.5 & 25.0 & 6.5 & 18.5 & 2.8 & 22.2 & .148 & 1.000 \\
\hline glandulosa & SE & 5.2 & 12.3 & 3.1 & 8.2 & 1.4 & 10.5 & & \\
\hline Opuntia ${ }^{3}$ & $\bar{x}$ & 316.7 & 169.4 & $40.2 \mathrm{~A}$ & $220.9 \mathrm{~B}$ & $46.7 \mathrm{~A}$ & 174.6 B & 2 & \\
\hline leptocaulis & SE & 5.9 & 27.8 & 17.9 & 24.8 & 21.0 & 20.1 & & \\
\hline Opuntia & $\bar{x}$ & 131.5 & 153.7 & 39.8 & 183.3 & 38.1 & 145.4 & .019 & .222 \\
\hline macrocentra & SE & 35.6 & 67.9 & 10.7 & 77.9 & 10.8 & 54.2 & & \\
\hline Opuntia ${ }^{3}$ & $\bar{x}$ & 388.9 & 193.5 & $97.3 \mathrm{~A}$ & $229.6 \mathrm{~A}$ & $141.9 \mathrm{~A}$ & $201.8 \mathrm{~A}$ & 2 & \\
\hline polyacantha & SE & 108.6 & 41.0 & 32.9 & 52.2 & 41.6 & 38.4 & & \\
\hline Yucca & $\bar{x}$ & 40.7 & 55.6 & 27.8 & 52.8 & 17.3 & 52.8 & .041 & .242 \\
\hline elata & SE & 16.9 & 23.0 & 18.3 & 21.6 & 11.1 & 23.4 & & \\
\hline
\end{tabular}

${ }^{1}$ Data rank transformed. Raw means presented.

${ }^{2}$ Treatment $\times$ time interaction $(P<.05)$. Means followed by same letter within years are not different $(P>.05)$.

${ }^{3}$ May 1995 results used as a covariate for postburn comparisons due to pre-treatment effects $(P<.10)$. Covariate adjusted means presented.

${ }^{4}$ Data square-root transformed. Raw means presented.

of variance because the distinct seasonal appearance of these species precluded direct comparison between the different sampling periods. We conducted all analyses using PROC GLM [23].

\section{Results}

4.1. Vegetation. Prescribed fire affected canopy coverage of the three major forage classes (Table $1 ; P<.05$ ). It reduced the canopy cover of shrubs and cacti to $66 \%$ of preburn cover.
Grass canopy cover was reduced to $16 \%$ of the preburn value immediately after the burn and recovered somewhat to $27 \%$ of preburn cover after one year. Forb canopy cover did not differ one month after treatment but increased 11-fold on the burned sites and was more than 4-fold greater than control sites at one year posttreatment.

The prescribed fire decreased stem density of threeshrub and two-cacti species (Table 2; $P<.05$ ). Tarbush and creosote bush densities were reduced by almost $80 \%$ on burned sites. Density of tasajillo and purple prickly pear was 
TABle 3: Mean frequency (\%) of grass species prior to burning, May 1995 (PRE), and one year post burn, July 1996 (POST96), on burned (B) and control (C) sites on Fort Bliss Military Reservation, Otero County, New Mexico.

\begin{tabular}{|c|c|c|c|c|c|c|c|}
\hline \multirow{2}{*}{ Species } & & \multicolumn{3}{|c|}{ PRE } & \multicolumn{3}{|c|}{ POST96 } \\
\hline & & B & $\mathrm{C}$ & $P$ & $\mathrm{C}$ & B & $P$ \\
\hline \multirow[t]{2}{*}{ Aristida spp. } & $\bar{x}$ & 1.6 & 2.6 & .525 & 16.8 & 1.3 & .124 \\
\hline & SE & 1.0 & 1.3 & & 8.8 & 1.0 & \\
\hline \multirow[t]{2}{*}{ Bouteloua barbata } & $\bar{x}$ & 0.0 & 0.0 & 1 & 17.6 & 6.6 & .121 \\
\hline & SE & 0.0 & 0.0 & & 6.7 & 4.4 & \\
\hline \multirow[t]{2}{*}{ Bouteloua eriopoda } & $\bar{x}$ & 8.0 & 2.3 & .361 & 11.6 & 3.6 & .073 \\
\hline & SE & 5.4 & 1.7 & & 6.6 & 3.3 & \\
\hline \multirow[t]{2}{*}{ Muhlenbergia arenacea } & $\bar{x}$ & 5.3 & 3.6 & .647 & 20.0 & 1.3 & .002 \\
\hline & SE & 2.8 & 1.8 & & 4.9 & 0.7 & \\
\hline \multirow[t]{2}{*}{ Muhlenbergia porteri } & $\bar{x}$ & 56.0 & 68.7 & .227 & 16.7 & 70.0 & $\leq .001$ \\
\hline & SE & 6.5 & 5.2 & & 3.1 & 5.6 & \\
\hline \multirow[t]{2}{*}{ Scleropogon brevifolius ${ }^{2}$} & $\bar{x}$ & 35.6 & 23.0 & .207 & 36.6 & 21.7 & .141 \\
\hline & SE & 10.8 & 9.8 & & 8.3 & 7.3 & \\
\hline \multirow[t]{2}{*}{ Sporobolus flexuosus } & $\bar{x}$ & 24.0 & 31.0 & .204 & 8.0 & 31.9 & $\leq .001$ \\
\hline & SE & 5.0 & 5.7 & & 2.7 & 5.1 & \\
\hline
\end{tabular}

${ }^{1}$ Statistical analysis not attempted.

${ }^{2}$ Data square-root transformed. Raw means presented.

$72 \%$ less after burning, while soaptree yucca density declined by almost half. We consider the $90 \%$ reduction in fourwing saltbush density biologically significant $(P=.075)$.

The prescribed fire altered frequency of three grass species one year posttreatment (Table 3; $P<.01$ ). Bush muhly and mesa dropseed (Sporobolus flexuosus) prevalence on burned sites was only $25 \%$ of control sites. Ear muhly responded favorably to the burn and was 15 times more frequent on the burned sites.

Frequency of occurrence of five forb species was altered by the prescribed fire treatment (Table $4 ; P<.01$ ). Broom snakeweed (Gutierrezia sarothrae) was the only species negatively impacted and was found only $8 \%$ as frequently on burned sites as on control sites. Leafy spurge (Euphorbia esula), trailing windmills (Allionia incarnate), hairyseed bahia (Bahia absinthifolia), and Gregg's heliotrope were at least seven times more frequent on burned sites than on controls.

4.2. Small Mammals. Thirty thousand trap-nights during 1995 and 1996 yielded 1744 captures of 766 individuals of 15 species. The burn did not affect species diversity or richness (Table 5). Four species had enough individual captures for valid extrapolation of fire effects: Chihuahuan Desert pocket mouse (Chaetodipus eremicus; $n=97$ ), Merriam's kangaroo rat (Dipodomys merriami; $n=436$ ), silky pocket mouse (Perognathus flavus; $n=163$ ), and plains pocket mouse ( $P$. flavescens; $n=42$ ). Limited captures (fewer than 20 individuals) of 11 species precluded statistical comparisons: Ord's kangaroo rat ( $D$. ordii), banner-tail kangaroo rat (D. spectabilis), white-throated woodrat (Neotoma leucodon), desert woodrat (N. micropus), Mearn's grasshopper mouse (Onychomys arenicola), northern grasshopper mouse $(O$. leucogaster), white-footed mouse (Peromyscus leucopus), deer mouse (P. maniculatus), western harvest mouse (Reithrodontomys megalotis), cotton rat (Sigmodon hispidus), and spotted ground squirrel (Spermophilus spilosoma).

The prescribed fire impacted relative abundance (minimum number of individuals alive within a 25 -ha study site) of three of the most prevalent small mammals (Table 5). Merriam's kangaroo rat relative abundance was unaffected one month after the prescribed fires but was 91\% greater on the burned sites than on controls one year posttreatment $(P<.01)$. Silky pocket mouse relative abundance was consistently affected by treatment, averaging 190\% greater on burned sites during both posttreatment sampling periods. Chihuahuan Desert pocket mice appeared to respond negatively to the fire, with relative abundance $170 \%$ greater on control sites than on burned sites $(P=.080)$. The plains pocket mouse and total relative abundance of Cricetidae and Heteromyidae did not vary between treatments.

\section{Discussion}

Reduced shrub canopy cover by prescribed burning coincides with past research in the southwestern deserts of North America [24, 25]. However, effects of fire, either prescribed or wild, on plant communities vary widely. Season of burn may have the greatest impact on resprouting and mortality of shrubs, especially creosote bush [24], one of the dominant shrubs on our sites. Wright and Bailey [1] suggested that June fires may kill $100 \%$ of creosote bush. However, on our study sites many creosote plants which were almost entirely burned off at the soil surface had resprouted one year postburn, indicating that June fires will not cause total mortality of creosote bush in the NCD. Density may be affected for a longer period of time because creosote bush seedling establishment is unpredictable, dependant on fall 
TABle 4: Mean frequency (\%) of forb species prior to burning, May 1995 (PRE), and one year post burn, July 1996 (POST96), on burned (B) and control (C) sites on Fort Bliss Military Reservation, Otero County, New Mexico.

\begin{tabular}{|c|c|c|c|c|c|c|c|}
\hline \multirow{2}{*}{ Species } & & \multicolumn{3}{|c|}{ PRE } & \multicolumn{3}{|c|}{ POST96 } \\
\hline & & $\mathrm{B}$ & $\mathrm{C}$ & $P$ & B & $\mathrm{C}$ & $P$ \\
\hline \multirow[t]{2}{*}{ Allionia incarnata } & $\bar{x}$ & 0.0 & 0.0 & 1 & 18.0 & 0.3 & .008 \\
\hline & SE & 0.0 & 0.0 & & 5.2 & 0.3 & \\
\hline \multirow[t]{2}{*}{ Bahia absinthifolia } & $\bar{x}$ & 0.0 & 0.0 & 1 & 12.7 & 0.7 & .027 \\
\hline & SE & 0.0 & 0.0 & & 4.8 & 0.4 & \\
\hline \multirow[t]{2}{*}{ Brassicaceae } & $\bar{x}$ & 8.2 & 4.1 & .564 & 0.0 & 0.0 & 1 \\
\hline & SE & 6.5 & 1.3 & & 0.0 & 0.0 & \\
\hline \multirow[t]{2}{*}{ Cryptantha albida } & $\bar{x}$ & 2.9 & 7.9 & .174 & 0.0 & 0.0 & 1 \\
\hline & SE & 0.9 & 3.0 & & 0.0 & 0.0 & \\
\hline \multirow[t]{2}{*}{ Euphorbia esula } & $\bar{x}$ & 0.0 & 0.0 & 1 & 58.6 & 7.9 & $\leq .001$ \\
\hline & SE & 0.0 & 0.0 & & 9.5 & 4.3 & \\
\hline \multirow[t]{2}{*}{ Gutierrezia sarothrae } & $\bar{x}$ & 6.3 & 15.6 & .042 & 0.7 & 9.3 & $\leq .001$ \\
\hline & SE & 2.3 & 3.6 & & 0.4 & 1.9 & \\
\hline \multirow[t]{2}{*}{ Heliotropium greggii } & $\bar{x}$ & 0.0 & 0.0 & 1 & 43.0 & 5.6 & .005 \\
\hline & $\mathrm{SE}$ & 0.0 & 0.0 & & 9.2 & 3.3 & \\
\hline \multirow[t]{2}{*}{ Solanum elaegnifolium } & $\bar{x}$ & 0.0 & 0.0 & 1 & 8.0 & 0.0 & .111 \\
\hline & SE & 0.0 & 0.0 & & 4.5 & 0.0 & \\
\hline
\end{tabular}

${ }^{1}$ Statistical analysis not attempted.

soil temperatures, and heavy fall precipitation [26]. Likewise, asexual reproduction following fires was not documented during our study or by Brown and Minnich [24].

The prescribed fire reduction in density of three prevalent cactus species may foretell of greater impacts over time. Thomas [27] suggested that fire damage to cacti and other succulents may be underestimated for up to three years. Damage to the outer phloem on the perimeter of the plant prevents distribution of photosynthesis products, while the xylem transports nutrient reserves and water from the roots to the apical meristem. Regrowth may be immediately evident, but eventual mortality is likely, especially for small plants. Additional mortality may result from insect damage, disease, or herbivory by rodents, lagomorphs, and domestic animals [1].

Although grass response to wild and prescribed fires in the Northern Chihuahuan and Sonoran Deserts has been documented by others $[7,28-31]$, those studies documented fire effects in different vegetative communities. The grass species of primary interest on our sites was black grama because of its continued decline in the region. Frequent, intense fires may damage black grama communities by causing long-term loss of perennial grass cover, potentially leading to increased erosion, desertification, and shrub encroachment [31], and Wright and Bailey [1] strongly suggest avoiding prescribed burns in black grama grasslands. Conversely, Valone et al. [32] did not detect any fire effects on black grama cover. The limitation of black grama to only 3 of our 10 blocks resulted in large variances and difficulty documenting potential fire effects. When analysis was performed on only those sites with black grama, the prescribed burns reduced canopy coverage [33]. Additionally, greater postburn perennial forb coverage may have temporarily suppressed black grama. Drought after fire may impede black grama recovery [34], although precipitation levels probably did not affect black grama during this study.

Bush muhly dominated all study sites prior to burning and was severely reduced by fire. Moreover, we did not observe any resprouting or seedling establishment during either postburn survey; only those plants surviving the burn were growing during 1996. Glendening [35] considered bush muhly particularly difficult to establish artificially, and our results suggest that bush muhly is severely harmed by June fires and will probably require long periods of time to return to preburn levels.

Unlike shrubs and grasses, fire effects on forbs have had little attention in the literature $[1,25,30]$. Only C. E. Bock and J. H. Bock [7, 25], Cornelius [31], and Drewa and Havsted [34] thoroughly examined forb responses in sacaton and black grama communities, respectively. In addition, Valone and Kelt [36] reported increased annuals after burns in Arizona's Chihuahaan Desert. These cases differ in species assemblages from our study sites, but one can draw generalizations from their results.

Forb communities may benefit from reduced grass competition following fire $[31,34,37]$. Fire may also benefit forbs through increased organic components [38] and available nitrogen [39]. C. E. Bock and J. H. Bock [7] found higher forb numbers on summer burned areas than on winter burned areas for two consecutive years. Cornelius [31] documented higher annual and perennial forb cover one year after burning; however, forb cover decreased dramatically by the third year. Conversely, C. E. Bock and J. H. Bock [25] documented a negative response to fire by forb species during the first year of a 2-year study in Arizona. They attributed negative effects to habitat, season, and fire intensity. 
TABLE 5: Relative abundance (minimum number alive per $25 \mathrm{ha}$ ) of small mammals, small mammal species richness, and Shannon's (H) and Simpson's (D) diversity indices prior to burning in May 1995 (PRE), one month post burn, July 1995 (POST-95), and one year post burn, July 1996 (POST-96), on 10 pairs of burned (B) and control (C) study sites on Fort Bliss Military Reservation, Otero County, New Mexico.

\begin{tabular}{|c|c|c|c|c|c|c|c|c|c|}
\hline & & \multicolumn{2}{|c|}{ PRE } & \multicolumn{2}{|c|}{ POST95 } & \multicolumn{2}{|c|}{ POST96 } & \multicolumn{2}{|c|}{$P$} \\
\hline & & $\mathrm{B}$ & $\mathrm{C}$ & $\mathrm{B}$ & $\mathrm{C}$ & $\mathrm{B}$ & $\mathrm{C}$ & Burn & Time \\
\hline Chaetodipus $^{1}$ & $\bar{x}$ & 1.5 & 1.1 & 1.6 & 2.6 & 1.0 & 1.9 & .080 & .480 \\
\hline eremicus & SE & 0.8 & 0.7 & 0.9 & 1.6 & 0.5 & 0.7 & & \\
\hline Dipodomys ${ }^{2}$ & $\bar{x}$ & 3.5 & 7.1 & $6.8 \mathrm{~A}$ & $6.0 \mathrm{~A}$ & $13.2 \mathrm{~A}$ & $6.9 \mathrm{~B}$ & 3 & \\
\hline merriami & SE & 1.1 & 1.2 & 1.1 & 1.2 & 1.6 & 2.0 & & \\
\hline Dipodomys & $\bar{x}$ & 0.2 & 0.1 & 0.0 & 0.0 & 0.2 & 0.1 & 4 & \\
\hline ordii & SE & 0.1 & 0.1 & 0.0 & 0.0 & 0.1 & 0.1 & & \\
\hline Dipodomys ${ }^{1}$ & $\bar{x}$ & 0.0 & 0.7 & 0.0 & 0.0 & 0.1 & 0.1 & 4 & \\
\hline spectabilis & SE & 0.0 & 0.5 & 0.0 & 0.0 & 0.1 & 0.1 & & \\
\hline Neotoma ${ }^{1}$ & $\bar{x}$ & 0.0 & 0.0 & 0.0 & 0.0 & 0.2 & 0.0 & 4 & \\
\hline albigula & SE & 0.0 & 0.0 & 0.0 & 0.0 & 0.2 & 0.0 & & \\
\hline Neotoma ${ }^{1}$ & $\bar{x}$ & 0.0 & 0.1 & 0.0 & 0.4 & 0.1 & 0.0 & 4 & \\
\hline micropus & SE & 0.0 & 0.1 & 0.0 & 0.2 & 0.1 & 0.0 & & \\
\hline Onychomys ${ }^{1}$ & $\bar{x}$ & 0.0 & 0.0 & 0.6 & 0.5 & 0.8 & 1.1 & 4 & \\
\hline arenicola & SE & 0.0 & 0.0 & 0.3 & 0.2 & 0.3 & 0.4 & & \\
\hline Onychomys ${ }^{1}$ & $\bar{x}$ & 0.1 & 0.1 & 0.1 & 0.0 & 0.0 & 0.1 & 4 & \\
\hline leucogaster & SE & 0.1 & 0.1 & 0.1 & 0.0 & 0.0 & 0.1 & & \\
\hline Perognathus ${ }^{2}$ & $\bar{x}$ & 2.3 & 0.9 & 6.2 & 2.4 & 4.5 & 1.4 & .043 & .261 \\
\hline flavus & SE & 0.7 & 0.3 & 1.8 & 1.1 & 1.3 & 0.5 & & \\
\hline Perognathus ${ }^{5}$ & $\bar{x}$ & 0.1 & 0.1 & 1.3 & 1.0 & 0.7 & 1.0 & .892 & .220 \\
\hline flavescens & SE & 0.1 & 0.1 & 0.3 & 0.3 & 0.3 & 0.4 & & \\
\hline Peromyscus ${ }^{1}$ & $\bar{x}$ & 0.0 & 0.2 & 0.1 & 0.0 & 0.0 & 0.0 & 4 & \\
\hline leucopus & SE & 0.0 & 0.1 & 0.1 & 0.0 & 0.0 & 0.0 & & \\
\hline Peromyscus $^{1}$ & $\bar{x}$ & 0.3 & 0.5 & 0.2 & 0.0 & 0.2 & 0.0 & 4 & \\
\hline maniculatus & SE & 0.2 & 0.2 & 0.1 & 0.0 & 0.1 & 0.0 & & \\
\hline Reithrodontomys & $\bar{x}$ & 2.9 & 2.1 & 0.1 & 0.8 & 0.2 & 0.0 & 4 & \\
\hline megalotis & SE & 0.3 & 0.7 & 0.1 & 0.3 & 0.1 & 0.0 & & \\
\hline Sigmodon 1 & $\bar{x}$ & 0.0 & 0.2 & 0.0 & 0.5 & 0.1 & 0.3 & 4 & \\
\hline hispidus & SE & 0.0 & 0.1 & 0.0 & 0.4 & 0.1 & 0.2 & & \\
\hline Spermophilus ${ }^{1}$ & $\bar{x}$ & 0.2 & 0.3 & 0.4 & 0.8 & 0.2 & 0.1 & 4 & \\
\hline spilosoma & SE & 0.1 & 0.2 & 0.2 & 0.4 & 0.1 & 0.1 & & \\
\hline \multirow[t]{2}{*}{ All individuals } & $\bar{x}$ & 11.0 & 13.5 & 15.7 & 16.5 & 20.1 & 14.7 & .479 & .520 \\
\hline & SE & 2.2 & 2.1 & 3.2 & 2.5 & 4.3 & 2.1 & & \\
\hline \multirow[t]{2}{*}{ Cricetidae $^{5}$} & $\bar{x}$ & 1.1 & 3.5 & $3.4 \mathrm{~A}$ & $3.0 \mathrm{~A}$ & $1.9 \mathrm{~A}$ & $1.4 \mathrm{~A}$ & 3 & \\
\hline & SE & 0.4 & 1.0 & 0.4 & 1.0 & 0.6 & 0.5 & & \\
\hline \multirow[t]{2}{*}{ Heteromyidae } & $\bar{x}$ & 7.6 & 10.0 & 14.4 & 13.5 & 18.2 & 13.0 & .268 & .423 \\
\hline & SE & 2.0 & 1.7 & 3.0 & 2.6 & 4.2 & 1.8 & & \\
\hline \multirow[t]{2}{*}{ Species richness } & $\bar{x}$ & 3.8 & 4.1 & 4.1 & 5.0 & 4.6 & 4.2 & .731 & .718 \\
\hline & SE & 0.3 & 0.4 & 0.5 & 0.4 & 0.7 & 0.6 & & \\
\hline \multirow[t]{2}{*}{$\mathrm{H}$} & $\bar{x}$ & 1.14 & 1.08 & 1.07 & 1.25 & 1.04 & 1.06 & .716 & .232 \\
\hline & SE & 0.10 & 0.12 & 0.08 & 0.09 & 0.10 & 0.11 & & \\
\hline \multirow[t]{2}{*}{$\mathrm{D}$} & $\bar{x}$ & 0.29 & 0.37 & 0.35 & 0.31 & 0.41 & 0.39 & .413 & .127 \\
\hline & SE & 0.03 & 0.05 & 0.03 & 0.03 & 0.06 & 0.05 & & \\
\hline
\end{tabular}

${ }^{1}$ Data rank transformed. Raw means presented.

${ }^{2}$ May 1995 results used as a covariate for postburn comparisons due to the potential for pre-treatment effects $(.05<P<.10)$. Postburn results are covariateadjusted means.

${ }^{3}$ Treatment $\times$ time interaction $(P<.05)$. Means followed by the same letter within years are not different $(P>.05)$.

${ }^{4}$ Limited captures ( $<20$ individuals) of this species precluded statistical comparisons.

${ }^{5}$ Data square-root transformed. Raw means presented. 
Small mammal population estimates based on probabilistic models are typically superior to those based on enumeration techniques [40]. In most instances, the number obtained from count statistics is likely to be smaller than the actual number of animals at a given study area [41]. In addition, when count statistics are used as indices of population size, sampling fraction estimations are typically not attempted, as standardized counting conditions have been assumed to yield sampling fraction estimates that are equal with regards to the populations being compared; however, sampling fractions often vary over time and space, neither of which can be easily controlled [41]. Furthermore, modeling capture probabilities has the advantage of being able to address variations in capture probabilities, such as those caused by time, behavioral responses, heterogeneity, and various combinations of these [42]. Unfortunately, the limited number of captured and recaptured animals in the present study did not fulfill statistical requirements for use of probabilistic models. The need to sample 20 study sites within a relatively short environmental season precluded longer trapping duration within sites, resulting in relatively low capture numbers. Because of this, we used an index of relative abundance (minimum number known alive) and focused on the same spatial locations and sampling time frames each year.

Changes in habitat composition and structure following disturbances may promote shifts in small mammal communities. Typically, researchers attribute community shifts to changes in microhabitats and food habits of individual species [3, 7, 15]. Lawrence [43] attributed loss of canopy cover to a shift in the community structure of chaparraladapted species to grassland-adapted species following a prescribed burn. C. E. Bock and J. H. Bock [7] also attributed change in the small mammal community to habitat changes in desert grassland in southeastern Arizona. In addition, researchers have suggested that fire-related fragmentation could increase predation pressure on small mammals [44].

Our findings of limited response to burning by the small mammal community indicated that either the habitat was not altered enough to facilitate community changes or not enough time had transpired for a response to occur. The significant changes documented within the vegetative community lead us to suggest that the latter is a more valid conclusion. However, a rodent community in the Sonoran Desert of California increased species diversity one year following a productivity pulse from the 1997-1998 El Nino event [45]. Orland and Kelt [45] related the lack of increase in small mammal diversity following an experimental resource pulse to the monopolization of the added resources by the largest pocket mouse (Chaetodipus formosus) present.

The relative response of fire-sensitive small mammals to patch burning was positively associated with greater rainfall in the Simpson Desert of Australia (Letnic and Dickman 2005). The almost 5 -fold increase in forb cover we documented one year after the prescribed fire should have provided abundant vegetative and seed sources for subsequent population responses. However, sporadic precipitation events in desert habitats require long-term studies of rodent populations to determine the lasting effects of fire on these communities. In some instances, fire has been thought to have large but delayed impacts on small mammal communities [46]. Even studies of long-term responses may falter, as Letnic et al. [2] could not array small mammal assemblages along a continuum of postfire vegetation succession.

Merriam's kangaroo rat was our most common species, and we expected the positive impact from the change in vegetative structure. C. E. Bock and J. H. Bock [7] found more heteromyids on burned areas than on unburned areas following summer and winter fires in a sacaton(Sporobolus sp.)-dominated habitat in southeastern Arizona. Other studies have linked low or decreased vegetative cover to increases in Dipodomys spp. [47-49]. Habitats opened up by fire may be favorable to kangaroo rats for foraging activities due to their predator avoidance adaptations [50].

Significant changes in the vegetative communities may have a long-term effect on small mammal populations. High volumes of seeds produced by winter and summer annuals and perennials on burned areas during years of aboveaverage precipitation may induce increased populations of small mammals. Conversely, several growing seasons with below-average precipitation following fires may cause small mammal populations to decline. Continued research on the long-term effects of fire on small mammals of the NCD is needed.

\section{Acknowledgments}

The authors thank the Army Research Office for financial support according to Research Agreement DAAH04-94-G0115. They thank the United States Army Construction Engineering Research Laboratories and the Natural Resources Branch, Directorate of Environment, Fort Bliss for logistical support, especially B. Russell, K. Von Finger, B. Locke, and T. Bashore. Access to the study sites was provided by the 1st Combined Arms Support Battalion, Fort Bliss. Additional thanks to Col. Lund, Capt. Kingery, Capt. Baker, G. Bankston, S. Offit, J. Brown, T. Johnson, and A. Warren for their assistance during site preparation and burning. At the time the research was conducted, S. Demarais was Associate Professor, Department of Range, Wildlife and Fisheries Management, Texas Tech University. This paper is contribution WF-313 of the Mississippi State University Forest \& Wildlife Research Center.

\section{References}

[1] H. A. Wright and A. W. Bailey, Fire Ecology: United States and Southern Canada, John Wiley \& Sons, New York, NY, USA, 1982.

[2] M. Letnic, C. R. Dickman, M. K. Tischler, B. Tamayo, and C.L. Beh, "The responses of small mammals and lizards to postfire succession and rainfall in arid Australia," Journal of Arid Environments, vol. 59, no. 1, pp. 85-114, 2004.

[3] W. O. Wirtz, D. Hoekman, J. R. Muhm, and S. L. Souza, "Postfire succession following prescribed fire in southeastern California chaparral," in Management of Amphibians, Reptiles, and Small Mammals in North America. United States Department of Agriculture, Forest Service General Technical Report 
RM-166, R. C. Szaro, K. E. Severson, and D. R. Patton, Eds., pp. 333-339, 1988.

[4] S. D. Thompson, "Structure and species composition of desert heteromyid rodent species assemblages: effects of a simple habitat manipulation," Ecology, vol. 63, no. 5, pp. 1313-1321, 1982.

[5] G. I. H. Kerley and W. G. Whitford, "Can kangaroo rat graminivory contribute to the persistence of desertified shrublands?" Journal of Arid Environments, vol. 73, no. 6-7, pp. 651657, 2009.

[6] D. P. Christian, "Effects of fire on small mammal populations in a desert grassland," Journal of Mammalogy, vol. 58, pp. 423427, 1977.

[7] C. E. Bock and J. H. Bock, "Response of birds, small mammals, and vegetation to burning sacaton grasslands in southeastern Arizona," Journal of Range Management, vol. 31, pp. 296-300, 1978.

[8] K. N. Geluso, G. D. Schroder, and T. B. Bragg, "Fire-avoidance behavior of meadow voles (Microtus pennsylvanicus)," American Midland Naturalist, vol. 116, no. 1, pp. 202-205, 1986.

[9] S. Legge, S. Murphy, J. Heathcote, E. Flaxman, J. Augusteyn, and M. Crossman, "The short-term effects of an extensive and high-intensity fire on vertebrates in the tropical savannas of the central Kimberley, northern Australia," Wildlife Research, vol. 35, no. 1, pp. 33-43, 2008.

[10] D. W. Kaufman, E. J. Fink, and G. A. Kaufman, "Small mammals and grassland fires," in Fire in North American Tallgrass Prairies, S. L. Collins and L. L. Wallace, Eds., pp. 4680, University of Oklahoma Press, Norman, Okla, USA, 1990.

[11] L. A. Brennan, R. T. Engstrom, and W. E. Palmer, "Whither wildlife without fire?" in Transactions of the 63rd North American Wildlife and Natural Resources Conference, vol. 63, pp. 402-414, Orlando, Fla, USA, March 1998.

[12] A. M. Penn, W. B. Sherwin, D. Lunney, and P. B. Banks, "The effects of a low-intensity fire on small mammals and lizards in a logged, burnt forest," Wildlife Research, vol. 30, no. 5, pp. 477-486, 2003.

[13] S. J. Converse, G. C. White, K. L. Farris, and S. Zack, "Small mammals and forest fuel reduction: national-scale responses to fire and fire surrogates," Ecological Applications, vol. 16, no. 5, pp. 1717-1729, 2006.

[14] M. E. Monroe and S. J. Converse, "The effects of early season and late season prescribed fires on small mammals in a Sierra Nevada mixed conifer forest," Forest Ecology and Management, vol. 236, no. 2-3, pp. 229-240, 2006.

[15] L. H. Simons, "Rodent dynamics in relation to fire in the Sonoran Desert," Journal of Mammalogy, vol. 72, no. 3, pp. 518-524, 1991.

[16] M. Letnic, B. Tamayo, and C. R. Dickman, "The responses of mammals to La Niña (El Niño Southern oscillation)associated rainfall, predation, and wildfire in central Australia," Journal of Mammalogy, vol. 86, no. 4, pp. 689-703, 2005.

[17] R. H. Schmidt, "Chihuahuan climate," in Proceedings of the 2nd Symposium on Resources of the Chihuahuan Desert Region, J. C. Barlow, A. M. Powell, and B. N. Timmermann, Eds., pp. 4063, Chihuahuan Desert Institute, 1986.

[18] C. D. Bonham, Measurements for Terrestrial Vegetation, John Wiley \& Sons, New York, NY, USA, 1989.

[19] E. D. Fleharty and K. W. Navo, "Irrigated cornfields as habitat for small mammals in the sandsage prairie of western Kansas," Journal of Mammalogy, vol. 64, pp. 367-379, 1983.

[20] W. Weaver and C. E. Shannon, The Mathematical Theory of Communication, University of Illinois, Urbana, Ill, USA, 1949.
[21] E. H. Simpson, "Measurement of diversity," Nature, vol. 163, no. 4148 , p. $688,1949$.

[22] W. J. Conover and R. L. Iman, "Rank transformations as a bridge between parametric and nonparametric statistics," The American Statistician, vol. 35, pp. 124-129, 1981.

[23] SAS Institute, SAS/STAT User's Guide. Release 6.03, SAS Institute, Cary, NC, USA, 1988.

[24] D. E. Brown and R. A. Minnich, "Fire and changes in creosote bush scrub of the western Sonoran Desert, California," American Midland Naturalist, vol. 116, no. 2, pp. 411-422, 1986.

[25] C. E. Bock and J. H. Bock, "Short-term reductions in plant densities following prescribed fire in an ungrazed semidesert shrub-grassland," The Southwestern Naturalist, vol. 37, pp. 4953, 1992.

[26] H. D. Grover and H. B. Musick, "Shrubland encroachment in southern New Mexico, U.S.A.: an analysis of desertification processes in the American Southwest," Climatic Change, vol. 17, pp. 305-330, 1990.

[27] P. A. Thomas, "Responses of succulents to fire: a review," Journal of Wildland Fire, vol. 1, pp. 11-22, 1991.

[28] D. R. Cable, "Fire effects on semidesert grasses and shrubs," Journal of Range Management, vol. 20, pp. 170-176, 1967.

[29] H. A. Wright, "The role and use of fire in the semidesert grassshrub type," Tech. Rep. INT 85, United States Department of Agriculture, Forest Service General, 1980.

[30] C. E. Bock and J. H. Bock., "Effects of fire on wildlife in southwestern lowland habitats," in Effects of Fire Management of Southwestern Natural Resources, J. S. Krammes, Ed., pp. 5064, United States Department of Agriculture, Forest Service General Technical Report RM-191, Fort Collins, Colo, USA, 1988.

[31] J. M. Cornelius, Fire effects on vegetation of a Northern Chihuahuan desert grassland, Dissertation, New Mexico State University, Las Cruces, NM, USA, 1988.

[32] T. J. Valone, S. E. Nordell, and S. K. M. Ernest, "Effects of fire and grazing on an arid grassland ecosystem," Southwestern Naturalist, vol. 47, no. 4, pp. 557-565, 2002.

[33] T. J. Monasmith, The effects of range fire on rodents at Fort Bliss, Texas, M.S. thesis, Texas Tech University, Lubbock, Tex, USA, 1997.

[34] P. B. Drewa and K. M. Havstad, "Effects of fire, grazing, and the presence of shrubs on Chihuahuan desert grasslands," Journal of Arid Environments, vol. 48, no. 4, pp. 429-443, 2001.

[35] G. E. Glendening, "Artificial revegetation of depleted semidesert ranges in southern Arizona. Progress Report, RR-SW investigative program, artificial revegetation," SRER Paper Archives file 549, College of Agriculture and Life Sciences, University of Arizona, Tucson, Ariz, USA, 1937.

[36] T. J. Valone and D. A. Kelt, "Fire and grazing in a shrubinvaded arid grassland community: independent or interactive ecological effects?" Journal of Arid Environments, vol. 42, no. 1, pp. 15-28, 1999.

[37] P. B. Drewa, D. P. C. Peters, and K. M. Havstad, "Fire, grazing, and honey mesquite invasion in black gramadominated grasslands of the Chihuahuan Desert: a synthesis," in Proceedings of the Invasive Species Workshop: The Role of Fire in the Control and Spread of Invasive Species, K. E. M. Galley and T. P. Wilson, Eds., Miscellaneous Publication no. 11, pp. 31-39, Tall Timbers Research Station, Tallahassee, Fla, USA, 2001.

[38] C. S. Crawford and J. R. Gosz, "Desert ecosystems: their resources in space and time," Environmental Conservation, vol. 9, no. 3, pp. 181-195, 1982. 
[39] J. M. Cornelius and G. L. Cunningham, "Nitrogen enrichment effects on vegetation of a northern Chihuahuan desert landscape," in Strategies for Classification and Management of Native Vegetation for Food Production in Arid Zones, W. Moir and E. Aldon, Eds., pp. 112-116, United States Department of Agriculture, Forest Service General Technical Report RM-150, 1987.

[40] J. D. Nichols and K. H. Pollock, "Estimation methodology in contemporary small mammal capture- recapture studies," Journal of Mammalogy, vol. 64, no. 2, pp. 253-260, 1983.

[41] J. D. Nichols, "Capture-recapture models. Using marked animals to study population dynamics," BioScience, vol. 42, pp. 94-120, 1952.

[42] G. C. White, D. R. Anderson, K. P. Burnham, and D. L. Otis, Capture-Recapture and Removal Methods for Sampling Closed Populations, Los Alamos National Lab, New Mexico, USA, 1982.

[43] G. E. Lawrence, "Ecology of vertebrate animals in relation to chaparral fire in the Sierra Nevada foothills," Ecology, vol. 47, pp. 278-291, 1966.

[44] I. Torre and M. Díaz, "Small mammal abundance in Mediterranean post-fire habitats: a role for predators?" Acta Oecologica, vol. 25, no. 3, pp. 137-142, 2004.

[45] M. C. Orland and D. A. Kelt, "Responses of a heteromyid rodent community to large- and small-scale resource pulses: diversity, abundance, and home-range dynamics," Journal of Mammalogy, vol. 88, no. 5, pp. 1280-1287, 2007.

[46] H. F. Recher, D. Lunney, and A. Matthews, "Small mammal populations in a eucalypt forest affected by fire and drought. I. Long-term patterns in an era of climate change," Wildlife Research, vol. 36, no. 2, pp. 143-158, 2009.

[47] M. L. Rosenzweig, "Habitat selection experiments with a pair of coexisting Heteromyid rodent species," Ecology, vol. 54, pp. 111-117, 1973.

[48] J. C. Beatley, "Environments of kangaroo rats (Dipodomys) and effects of environmental change on populations in southern Nevada," Journal of Mammalogy, vol. 57, no. 1, pp. 67-93, 1976.

[49] B. Hayward, E. J. Heske, and C. W. Painter, "Effects of livestock grazing on small mammals at a desert cienega," Journal of Wildlife Management, vol. 61, pp. 123-129, 1997.

[50] B. P. Kotler, "Risk of predation and the structure of desert rodent communities," Ecology, vol. 65, no. 3, pp. 689-701, 1984. 

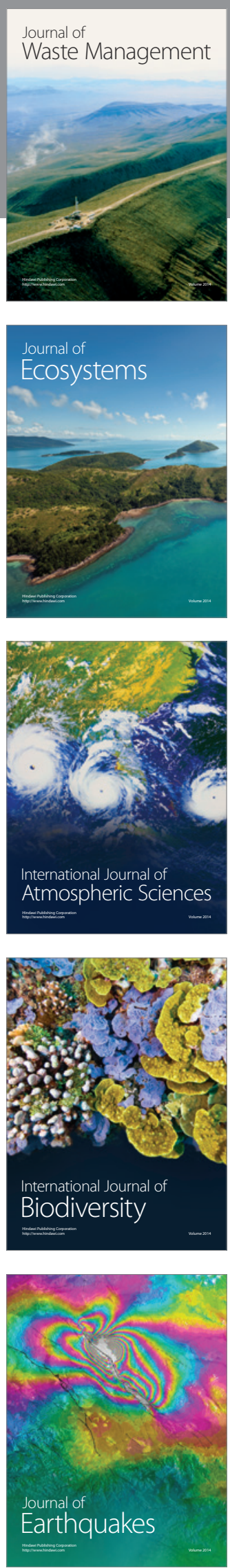
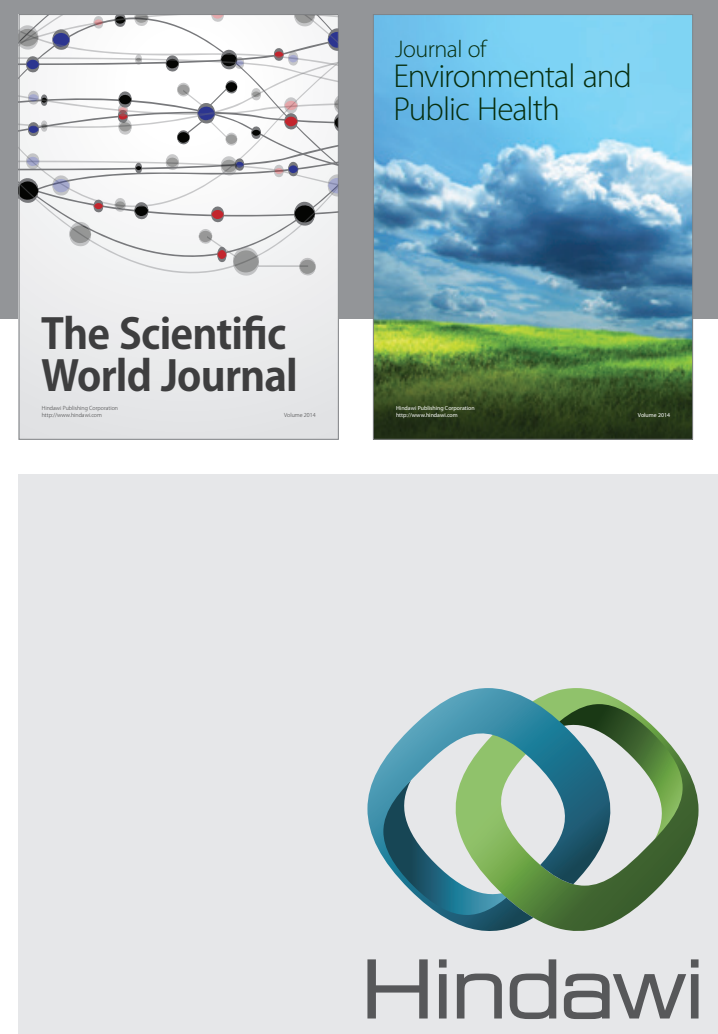

Submit your manuscripts at

http://www.hindawi.com
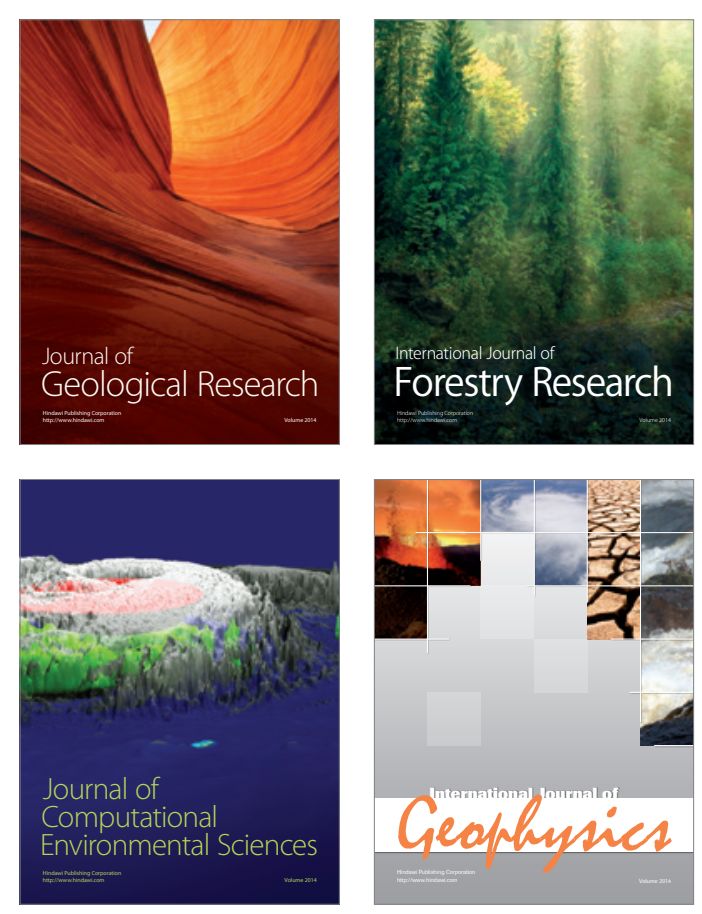
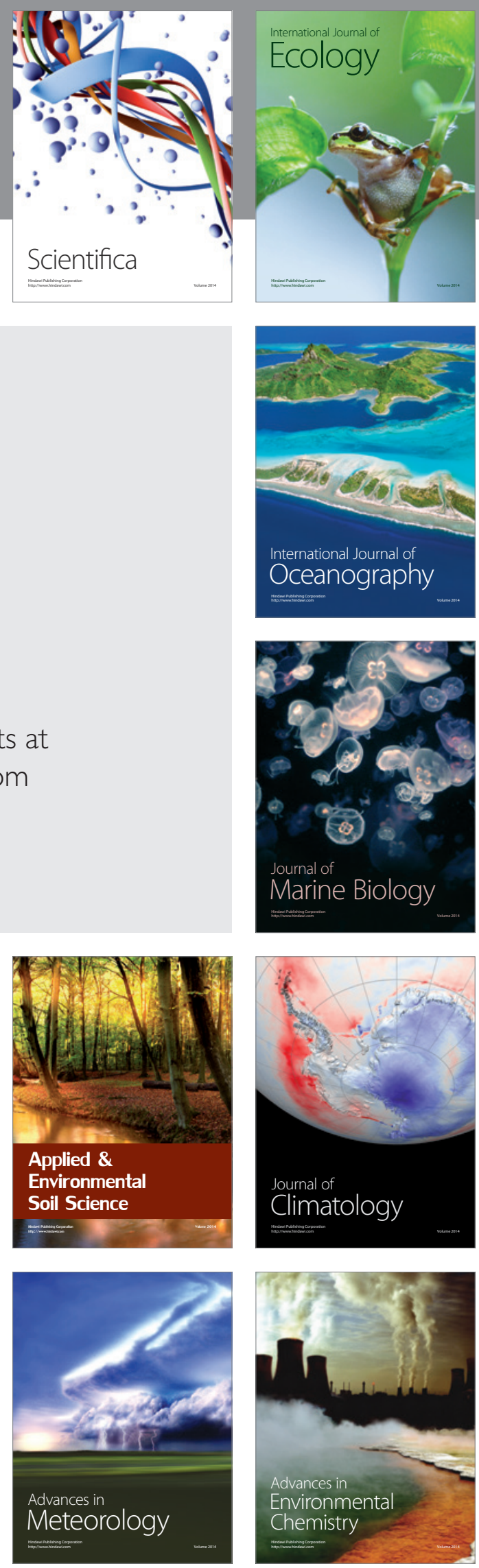